\title{
EVALUAR Y CALIFICAR EN PLÁSTICA. UNA EXPERIENCIA DE ÉXITO EN EDUCACIÓN PRIMARIA
}

\author{
Assess and grade in Art. An experience of success in Primary Education \\ Avaliar e classificar em artes plásticas. Uma experiência de sucesso no ensino primário \\ Aroa Mediero González (1) \\ (1) Universidad de Valladolid, España. Correo electrónico: aroamego@hotmail.com
}

\section{Resumen}

El trabajo presenta una experiencia de éxito puesta en práctica en dos clases de $6^{\circ}$ curso de Educación Primaria en el área de Plástica. Ante la dificultad para evaluar y calificar en esta asignatura, se ha realizado un estudio en el que se han manejado instrumentos para evaluar y calificar a los alumnos a lo largo de dos trimestres. El estudio muestra los instrumentos empleados por el docente y principalmente por el alumnado en este proceso: diarios y rúbricas para la evaluación y escalas graduadas para la calificación. El resultado del estudio muestra la utilidad y beneficio de estos instrumentos en las clases de Plástica.

Palabras clave: Educación Artística; evaluación formativa y compartida; investigación Acción; innovación docente; evaluación para el aprendizaje

\footnotetext{
Abstract

This paper presents an experience put into practice with two classes of $6^{\text {th }}$ course of Primary Education in the subject of art. Due to the difficulty to evaluate and grade in the subject of art, it has conducted a study which shows instruments to assess and grade students along two terms. The study shows the instruments adopted by the teacher and mainly by the students during this process: diaries and rubrics in the process of assessment and graduated scales in the rating process. The results of the research show the usefulness and benefit of these instruments in the lessons of art.

Keywords: Art Education; formative and shared assessment; action-research; educational innovation; assessment for learning
} 


\section{Resumo}

Este trabalho apresenta uma experiência de sucesso, posta em prática em duas turmas do 6. ${ }^{\circ}$ ano do ensino básico na disciplina de artes plásticas. Face à dificuldade em avaliar e classificar nesta disciplina, realizou-se um estudo em que se utilizaram instrumentos para avaliar e classificar os alunos ao longo de dois trimestres. $\mathrm{O}$ estudo mostra os instrumentos utilizados pelo professor e, principalmente, pelos alunos neste processo: diários e rubricas para a avaliação e escalas graduadas para a classificação. O resultado do estudo mostra a utilidade e benefício destes instrumentos nas aulas de artes plásticas. Palavras-chaves: Educação Artística; avaliação formativa e partilhada; pesquisa-ação; inovação docente; avaliação para a aprendizagem

\section{Introducción}

Un sistema de evaluación y calificación apropiado y beneficioso para el alumnado y para el docente es necesario en todo proceso de aprendizaje (López-Pastor 2015, 2017). En Plástica este proceso suele estar en segundo plano siendo necesario la creación de técnicas e instrumentos que faciliten esta labor (Sánchez, 2014). Esta experiencia presenta instrumentos manejados que son efectivos en la mejora de las calificaciones.

\section{Contextualización}

En el curso 2018/2019, hemos puesto en práctica un nuevo modelo de evaluación y calificación en dos grupos de $6^{\circ}$ de Educación Primaria de 17 y 18 alumnos de un centro público ubicado en un entorno rural con elevado número de alumnos. El área en el que se ha centrado nuestra práctica es Educación Artística y, más específicamente, en Plástica.

En cursos previos ya realizamos una serie de ciclos de investigación-acción en esta área que nos permitieron desarrollar una metodología centrada en el arte contemporáneo, junto con un nuevo sistema de evaluación/calificación. En función de ese estudio hemos desarrollado esta experiencia a lo largo del curso poniendo en práctica este modelo en cada unidad.

\section{Diseño y desarrollo}

Gracias al manejo de diarios y rúbricas conseguimos desarrollar sistemas de evaluación formativa y compartida entre docentes y alumnado. 
-Sistema de evaluación formativa

Los instrumentos de evaluación manejados son:

- Diarios

Los alumnos se evalúan al finalizar cada unidad. Responden a cuestiones del docente sobre el trabajo realizado y lo aprendido. Posteriormente el maestro lo recoge y hace anotaciones proporcionando feedback y ofreciendo posibilidades de mejora.

- Rúbricas del alumno

Cada unidad tiene su rúbrica y es entregada una copia al inicio de la misma a cada alumno.

Cada indicador que aparece en la rúbrica son los aspectos que se tienen en cuenta para evaluar su práctica. Además, es el documento a partir del que se dará el salto a la calificación trimestral.

Finalizadas las prácticas, el alumnado entrega el diario con la rúbrica cumplimentada marcando dónde consideran que se encuentran en cada indicador. Así, el docente conoce si son conscientes de sus progresos. De igual manera, permite observar si saben realizar su autoevaluación coherentemente. Si existe discordancia entre la opinión del alumno y del docente se habla con los estudiantes para alcanzar posturas conciliadoras.

- Rúbricas del docente

El maestro tiene una rúbrica por cada alumno en cada trimestre siguiendo el mismo modelo que la que tienen ellos.

En ella anota por cada unidad en qué estado está cada alumno. De este modo, permite observar su progresión en el trimestre, si se ha producido una evaluación continua positiva. En definitiva, si se ha producido evaluación formativa del alumnado a partir del manejo de rúbricas y diarios y si existe correspondencia entre la opinión del docente y el alumnado con el fin de establecer o no un proceso dialógico.

-Sistema de calificación.

Los grupos conocen el sistema de evaluación y calificación al inicio de curso contribuyendo así en su aprendizaje puesto que saben qué hacer para lograr calificaciones elevadas. 
Importante desafío al que se enfrenta el docente es dar el salto a la calificación. La evaluación formativa realizada a lo largo del curso permite dar este salto gracias a la rúbrica. El sistema de calificación establecido tiene su origen en ese instrumento, existiendo una clara coherencia entre las categorías de la rúbrica con los criterios de una escala graduada. Este instrumento expone los criterios para lograr la calificación detallada en la misma. Asignar unos principios a cada grado de calificación facilita el trabajo al alumnado y al docente para establecer una calificación coherente.

$\mathrm{Su}$ desarrollo horizontal facilita a los alumnos a focalizar su aprendizaje si pretende obtener calificaciones elevadas.

Al finalizar cada trimestre los estudiantes reflejan su parecer en cuanto a su calificación. Conociendo el docente la valoración de los estudiantes plantea la calificación. Si existe disonancia entre ambos se lleva a cabo una conversación dialógica entre las partes con el objetivo de alcanzar una calificación ecuánime.

La Tabla 1 evidencia que, de manera general, los resultados en las calificaciones del alumnado han mejorado. En ella observamos que el número de suficientes aumentó debido a que los alumnos no entregaron ni el diario con las preguntas y la rúbrica ni su escala graduada, de ahí la calificación.

Tabla 1.

Resultados académicos

\begin{tabular}{lcccc}
\hline & \multicolumn{2}{c}{$\begin{array}{l}\mathbf{N}^{\mathbf{0}} \\
\text { Porcentaje } \\
\text { Calificación }\end{array}$} & $\begin{array}{l}\mathbf{1}^{\mathbf{a}} \text { Evaluación } \\
\text { Evas }\end{array}$ & $\begin{array}{l}\mathbf{N}^{\mathbf{2}} \\
\text { Porcentaje } \\
\mathbf{2}^{\mathbf{a}} \text { Evaluación }\end{array}$ \\
\hline Sobresaliente & $2,9 \%$ & 1 & $20 \%$ & 7 \\
Notable & $55,9 \%$ & 19 & $51,4 \%$ & 18 \\
Bien & $38,2 \%$ & 13 & $14,3 \%$ & 5 \\
Suficiente & $2,9 \%$ & 1 & $14,3 \%$ & 5 \\
Suspenso & $0 \%$ & 0 & $0 \%$ & 0 \\
Totales & $\mathbf{1 0 0}$ & $\mathbf{3 4}$ & $\mathbf{1 0 0}$ & $\mathbf{3 5}$ \\
\hline
\end{tabular}

Es destacable cómo el porcentaje de sobresalientes aumentó, procediendo de alumnos con bien y notable en la primera evaluación.

Además, de manera general, los alumnos mejoraron sus calificaciones.

\section{Evaluación y conclusiones}

La conveniencia del manejo de estos instrumentos se refleja en la mejora en las calificaciones (Tabla 1). Gracias a los instrumentos empleados observamos cómo evolucionan en el uso de conceptos artísticos en sus diarios, así como en sus creaciones 
plásticas. Con los instrumentos manejados los estudiantes son conocedores de lo que se va a tener en cuenta en los procesos de evaluación y posterior calificación. En todo este proceso es fundamental el feedback del docente en sus documentos y en los procesos dialógicos generados.

Al tratarse del tercer año empleando estos instrumentos, nuestra confianza en su uso revierte en beneficio del alumnado. Su manejo con diferentes grupos y niveles pone de manifiesto su conveniencia en el aula de Plástica (Mediero, 2018).

Al igual que sucedió años anteriores, el principal inconveniente que hallamos es la contrariedad por parte de algunos alumnos ante el trabajo que supone (Mediero, 2018). Tradicionalmente la Educación Artística no está asociada con el trabajo que proponemos por lo que puede generar rechazo al inicio, pero esta oposición se supera cuando entienden su validez.

Tras varios años manejando este sistema, podemos concluir que verdaderamente es conveniente y provechoso su manejo por alumnado y docentes puesto que genera una evaluación que favorece el aprendizaje además de facilitar el salto a la calificación.

\section{Referencias}

López-Pastor V. M., \& Pérez-Pueyo, A. (Coords.) (2017). Evaluación formativa y compartida en Educación: experiencias de éxito en todas las etapas educativas. León: Universidad de León. Recuperado de http://buleria.unileon.es/handle/10612/5999

López-Pastor, V. M. (2011). El papel de la evaluación formativa en la evaluación por competencias: aportaciones de la red de evaluación formativa y compartida en docencia universitaria. REDU. Revista de Docencia Universitaria, 9(1), 159173. doi: 10.4995/redu.2011.6185.

Mediero, A. (2018). ¿Cómo evaluar y calificar en el área de Plástica? Una experiencia de éxito en Educación Primaria. XI Congreso Nacional de Evaluación formativa y compartida. La evaluación educativa: entre la emoción y la razón. Córdoba. (En prensa)

Sánchez, L. (2014). Evaluación creativa: estrategias contemporáneas para representar el aprendizaje en la Educación Artística en la ESO (Tesis Doctoral). Universidad Complutense, Madrid. 
Tabla 2.

Modelo de rúbrica. Se adapta en cada práctica en función de los contenidos

\begin{tabular}{|c|c|c|c|c|}
\hline & Genio & Artista & Aprendiz & Ejecutor \\
\hline 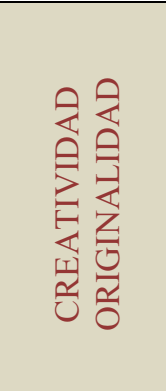 & $\begin{array}{l}\text { El trabajo muestra gran } \\
\text { atención en la realización. } \\
\text { Todos los elementos reflejan } \\
\text { alto grado de } \\
\text { personalización/originalidad. } \\
\text { La creación muestra riesgos y } \\
\text { alternativas a las ideas del } \\
\text { profesor/artista/compañeros. } \\
\text { Es un trabajo del alumno. }\end{array}$ & $\begin{array}{l}\text { El trabajo muestra } \\
\text { algún rasgo de } \\
\text { personalización/ } \\
\text { originalidad. } \\
\text { Algún elemento de la } \\
\text { creación no muestra } \\
\text { especial cuidado. }\end{array}$ & $\begin{array}{l}\text { Hay un elemento que } \\
\text { personaliza en el trabajo. } \\
\text { La idea no es creativa, es } \\
\text { típica de lo que puede } \\
\text { esperarse. } \\
\text { No hay cuidado en la } \\
\text { realización. La obra es más } \\
\text { bien una copia del } \\
\text { profesor/artista/compañero }\end{array}$ & $\begin{array}{l}\text { El trabajo no } \\
\text { muestra la } \\
\text { personalización de } \\
\text { ningún elemento. } \\
\text { Es una copia del } \\
\text { profesor/artista/ } \\
\text { compañeros. }\end{array}$ \\
\hline 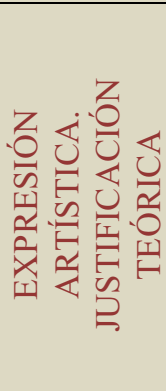 & $\begin{array}{l}\text { Puede definir los términos } \\
\text { trabajados explicándolo con } \\
\text { claridad. Realiza valoraciones } \\
\text { personales estableciendo } \\
\text { diferencias. } \\
\text { Comprende el origen y } \\
\text { evolución de lo trabajado. }\end{array}$ & $\begin{array}{lrr}\begin{array}{l}\text { Define más } \\
\text { término de }\end{array} & \text { un } \\
\text { Maneja } & \text { no } & \text { muy } \\
\text { seguro } & \text { en } & \text { qué } \\
\text { consisten. } & & \\
\text { La } & & \\
\text { personal valoración } \\
\text { totalmente no } & & \text { está } \\
\text { justificada. } & & \end{array}$ & $\begin{array}{l}\text { Define el término principal } \\
\text { manifestando } \\
\text { escuetamente en qué } \\
\text { consiste y en qué se } \\
\text { diferencia de otros. } \\
\text { Le cuesta hacer } \\
\text { manifestaciones } \\
\text { personales. }\end{array}$ & $\begin{array}{l}\text { Tiene dificultad en } \\
\text { definir el término y } \\
\text { le cuesta describirlo. } \\
\text { No hace } \\
\text { valoraciones } \\
\text { personales. }\end{array}$ \\
\hline 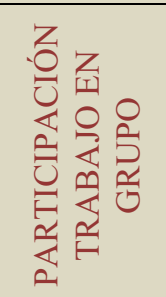 & $\begin{array}{l}\text { Ayuda a mantener el grupo } \\
\text { concentrado en las actividades } \\
\text { y sus aportaciones son } \\
\text { significativas. Trata de } \\
\text { mantener la unión de los } \\
\text { miembros trabajando en } \\
\text { grupo. }\end{array}$ & $\begin{array}{lr}\text { Participa en } & \text { las } \\
\text { actividades } & y \\
\text { contribuye } & \text { con } \\
\text { alguna sugerencia. } \\
\text { No causa problemas } \\
\text { en el grupo. }\end{array}$ & $\begin{array}{l}\text { A veces participa. } \\
\text { No distrae al grupo, pero } \\
\text { en ocasiones no es un buen } \\
\text { miembro. }\end{array}$ & $\begin{array}{l}\text { No participa en el } \\
\text { trabajo y distrae a } \\
\text { los compañeros. No } \\
\text { es buen miembro } \\
\text { del grupo y no } \\
\text { apoya el esfuerzo de } \\
\text { su grupo. }\end{array}$ \\
\hline 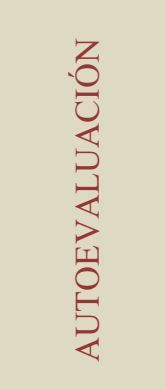 & $\begin{array}{l}\text { Usa criterios variados para } \\
\text { valorar su trabajo: expresión, } \\
\text { ideas, creatividad... } \\
\text { Nombra estrategias que ha } \\
\text { usado y analiza críticamente } \\
\text { su creación usando términos } \\
\text { apropiados. }\end{array}$ & $\begin{array}{l}\text { Usa algún criterio } \\
\text { para valorar su } \\
\text { trabajo. } \\
\text { Nombra alguna } \\
\text { estrategia usada y } \\
\text { analiza básicamente } \\
\text { su creación con } \\
\text { terminología } \\
\text { adecuada. }\end{array}$ & $\begin{array}{l}\text { Trata de usar criterios } \\
\text { estéticos para juzgar la } \\
\text { obra realizada pero no lo } \\
\text { hace adecuadamente. } \\
\text { No es capaz de realizar } \\
\text { una valoración crítica de, } \\
\text { aunque lo intenta. }\end{array}$ & $\begin{array}{l}\text { Evalúa su trabajo } \\
\text { como bueno/malo } \\
\text { basándose en su } \\
\text { gusto personal. } \\
\text { No establece } \\
\begin{array}{l}\text { relación con los } \\
\text { conceptos }\end{array} \\
\begin{array}{l}\text { trabajados en } \\
\text { aula. el }\end{array}\end{array}$ \\
\hline 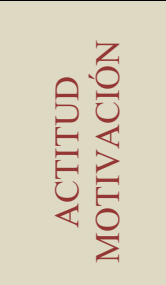 & $\begin{array}{l}\text { Absoluta implicación en el } \\
\text { trabajo. Muestra gran interés } \\
\text { por saber más. Usa los nuevos } \\
\text { conocimientos en otras áreas. } \\
\text { Comparte } \\
\text { relacionadas noticias } \\
\text { comentarios espontáneos. hace }\end{array}$ & $\begin{array}{l}\text { Gran implicación en } \\
\text { el trabajo. Actitud } \\
\text { positiva e interés por } \\
\text { saber más. Hace } \\
\text { algún comentario } \\
\text { sobre el tema en otro } \\
\text { contexto. }\end{array}$ & $\begin{array}{l}\text { Muestra interés el trabajo, } \\
\text { pero no hay deseo por } \\
\text { conocer más. } \\
\text { No realiza comentarios } \\
\text { espontáneos sobre el tema. }\end{array}$ & \begin{tabular}{l}
\multicolumn{2}{l}{ Exclusivamente } \\
realiza el trabajo \\
propuesto $r \quad$ sin \\
mostrar ningún tipo \\
de interés o deseo \\
por el \\
trabajado.
\end{tabular} \\
\hline
\end{tabular}


Tabla 3.

Escala graduada área de plástica.

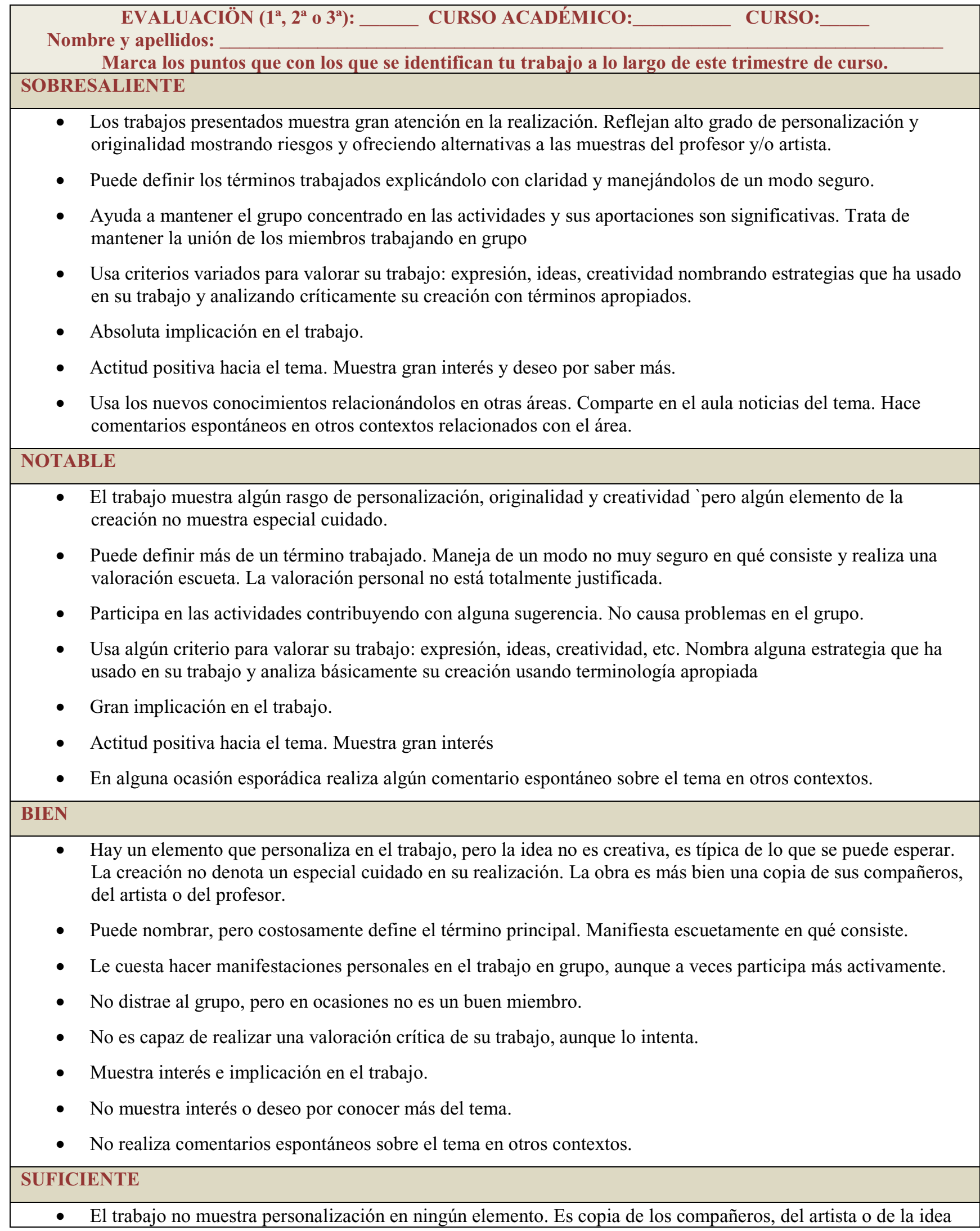




- Del profesor.
- Nificultad en definir los términos trabajados. Le cuesta justificar en qué se diferencian de otros.
- No participa activamente en el trabajo y/o distrae a los compañeros.
- No es buen miembro del grupo. Hay alguna actitud negativa que causa problemas en su grupo o en la clase.
- Sus palabras no tienen ningún tipo de relación con los conceptos trabajados en el aula.
- Exclusivamente realiza el trabajo propuesto sin mostrar ningún tipo de interés o deseo por el tema trabajado.
NO APRUEBA
- No ha realizado//entregado todos los trabajos.
- Evalúa su trabajo como bueno o malo basándose en su gusto personal.
- No participa correctamente en el grupo y en muchas ocasiones muestra comentarios o actitudes que influyen
naramente escucha, comparte o apoya el esfuerzo de su equipo.

